\title{
Use of soil streptomycetes from Maseno (Kenya) to inhibit growth of Pyricularia grisea invitro cultures.
}

\author{
Opande George ${ }^{1}$, Were Okumu ${ }^{2}$ and Arama Peter ${ }^{3}$. \\ ${ }^{\prime}$ (Department of Botany, Maseno University, Private Bag, Maseno. Kenya.) \\ ${ }^{2}$ (Department of Botany, Maseno University, Private Bag, Maseno Kenya.) \\ 3 (Department of App. Sci. Maseno University, Private Bag' Maseno. Kenya.)
}

\begin{abstract}
The most serious biotic constraint to finger millet worldwide is blast caused by the fungus Pyricularia grisea. Its management has been very challenging. However not a formidable control solution has been offered and therefore a need for other approachs. Actinomycetes belong to the phylum proteobacteria, they are gram $+v e$, terrestrial or aquatic with high guanine and cytosine content. Soil actinomycetes have antagonistic activity against a wide range of plant pathogens. This study isolated and characterizes some soil actinomycetes in Maseno area (Western Kenya) and studied their antifungal effect on P. grisea pathogenic on finger millet. Soil sample collected was dissolved in distilled water, homogenized and spread over modified Kuster's agar. Colonies of actiomycetes were isolated as from the fourth day of incubation. . Finger millet heads and leaves displaying symptoms of blast were collected and Pyricularia grisea isolated on autoclaved powered finger-millet debris and maintained on PDA. A single steak of actinomycetes culture was made on a fresh Kuster's agar and incubated for $3-4$ days at $26^{\circ} \mathrm{C}-28^{\circ} \mathrm{C}$, and P. grisea, $20 \mathrm{~mm}$, at right angles to the original streak of actinomycetes. The results indicate that isolates AT2 and AT4 are pathogenic to Pyricularia grisea and can be a basis for bio-control of blast in finger millet.
\end{abstract}

Keywords: Finger milet, actinomycetes, pyricularia, proteobacteria, kuster's agar, biopesticide

\section{Introduction}

The most serious constraints in finger millet production are those related to productivity enhancement (Oduori et al. 2007). ). Blast caused by Pyricularia grisea has been identified as the highest priority constraint to finger millet production in East Africa, (Takan et al. 2004). It is especially serious in the Busia, and Kisii counties of Kenya and in the main finger millet areas in north and east Uganda (Mgonja et al. 2007). Coming up with varieties that are resistant to blast disease has been a problem (Screenivaprasad et al. 2007). Blast affects finger millet at all growth stages, from seedling stage (causing lesions and premature drying of young leaves) to affecting the panicle causing neck and/or finger blast (Talbolt. 2003). Control of plant diseases has depended primarily upon the application of chemical fungicides, despite potentially toxic effects on humans, wildlife, and the environment (Hong-Sik et al. 2000). The advent of new pathogen races that are resistant to fungicides has been another problem to plant pathologists and agro chemists (in rice blast). Therefore, new control strategies that are safe to humans and wildlife and have benign environmental profiles, low use rates, and high specificity to target organisms are needed to ensure stable crop production (Arifuzzaman et al. 2010). Micro-organisms and their natural products are potentially important for the biological control of crop diseases without detrimental effects to the environment (Nonoh et al. 2010). Soil actinomyces have revealed their wide antifungal activity (Tinatin et al. 2006). The predominant species of actinomyces is streptomyces. Streptomyces are among the most producers of biologically active substances such as vitamins, alkaloids, plant growth factors, enzymes and enzyme inhibitors (Zarandi et al. 2009). Soil Streptomycetes are major contributors to the biological buffering of soils and have roles in decomposition of organic matter conducive to crop production (Dastager et al. 2006). Streptomyces species and a few other actionmycetes have been shown to protect several different plants to various degrees from soil-borne fungal pathogens (Reddi and Rao 1971). Streptomyces rochei and Streptomyces rimosus from the chickpea rhizosphere were found to be strong antagonists of Fusarium oxysporum f. sp. ciceri. Streptomyces hygroscopicus var. geldanus, grown in sterile soil, antagonized Rhizoctonia solani, the pea rootrot fungus, via geldanamycin production (Rothrock and Gottlieb 1984). Studies even show that use of Streptomycetes enhances growth of the crop plants. The objectives of this study were to isolate and characterize actinomycetes strains collected from Maseno soils and also to investigate their atagonistic effects on Pyricularia grisea cultures in vitro. 


\section{Materials And Methods}

\subsection{Soil sample collection and isolation of actinomycetes}

Stratified random sampling method was used to collect soil samples from agricultural lands in Maseno division. Samples were collected along a gradient (rural access roads) in Maseno region at $0.5 \mathrm{~km}$ interval. At each location was collected from top $4 \mathrm{~cm}$ soil profile and placed in sterile polythene bags. Samples were then stored at $-40^{\circ} \mathrm{C}$. Isolation of actinomycetes was carried out following the methods described by Baskaran et al (2010). One gram of soil sample was dissolved in $10 \mathrm{ml}$ sterile distilled water in a test-tube. The sample was homogenized and then left to stand for one hour. The test tubes were immersed in a water bath at $75{ }^{\circ} \mathrm{C}$ for 15 minutes then allowed to cool before plating.

\subsection{Isolation of Streptomycetes}

About $0.1 \mathrm{ml}$ of the prepared soil sample was spread over modified Kuster's gar medium (Baskaran et al. 2010) g/L: Starch 10, glycerol 0.3, $\mathrm{KNO}_{3} 3, \mathrm{~K}_{2} \mathrm{HPO}_{4} 2, \mathrm{NaCl} 2$, and $\mathrm{MgSO} 40.05, \mathrm{CaCO}_{3} 0.02, \mathrm{FeSO}_{4} 0.01$, agar 16; $\mathrm{pH} 7.0 \pm 0.1$. Culture plates incubated at $22-25^{\circ} \mathrm{C}$ and observed every day for growth. Growing cultures were re- isolated and plated onto fresh medium (Baskaran et al. 2010). A small portion of typical isolated colonies was streaked on Kuster's agar media and incubated at $25^{\circ} \mathrm{C}$ for $4-7$ days. All the morphologically different actinomycetes colonies were sub-cultured on Kuster's agar medium and maintained in the same for further investigations.

\subsection{Collection, isolation and Culture of Pyricularia grisea}

Collection of diseased plants from finger- millet growing regions was carried out during the humid and hot months of October- November 2011. The samples were dried under shade and preserved within the laboratory in closed cabinets. The leaves and heads showing diseased lesions were cut into pieces, $0.5 \mathrm{~cm} \mathrm{x} 0.5$ $\mathrm{cm}$ long whilst ensuring the inclusion of diseases and healthy tissue. The pieces were surface sterilized by dipping in $2.5 \%$ Sodium hypochlorite for one minute followed by rinsing in sterile water and then dipped in $70 \%$ ethanol for 30 seconds. Tissues were then rinsed in sterile water, dried on filter paper and then plated on finger-millet agar medium. The medium constituted $20 \mathrm{gm}$ finger-millet grain powder, $15.0 \mathrm{gm}$ agar dissolved in $1000 \mathrm{ml}$ tap water. The medium was autoclaved at $121^{\circ} \mathrm{C}$ for $15 \mathrm{~min}$. Developing cultures were isolated and plated onto fresh PDA medium.

\subsection{In vitro antifungal bioassays}

Antifungal bioassays were carried out according to the methods described by (Arifuzzaman et al. 2010 and Zarandi et al. 2009) with some modifications. A single steak of actinomycetes was made on fresh Kuster's agar and incubated for 3-4 days at $26^{\circ} \mathrm{C}-28^{\circ} \mathrm{C}$, after observing a good ribbon like growth of actinomyces, the fungal pathogen was also streaked, $20 \mathrm{~mm}$, at right angle to the original streak of actinomyces. The inhibition zone $(\mathrm{mm})$ was measured at a time interval of 24 hours. A total of six observations were made. During incubation, growth or lack of growth of the fungus was investigated both visually and microscopically. Control plates were maintained without inoculating actinomyces/fungus to assess the normal growth of the pathogenic fungi and actinomyces. Antifungal activity around the actinomyces agar plates was assessed following the methods described by Zarandi et al.(2010); (1) no inhibition = mycelial growth not different from control (0-4 $\mathrm{mm}$ ); (2) weak inhibition = partial inhibition of mycelia growth, measured as a diameter of 5-9 $\mathrm{mm}$; (3) moderate inhibition $=$ almost complete inhibition of mycelial growth, measured as a diameter of $10-19 \mathrm{~mm} ; 4$ ) strong inhibition $=$ complete inhibition, in which most mycelia did not grow, measured as a diameter of $20 \mathrm{~mm}$.

\subsection{Actinomycetes isolated.}

\section{Results And Discussions}

There was a high diversity of actinobacterial isolates in soils from Maseno. This finding is also in line with what Nonoh et al. (2010) found in their study of Western Kenya. Isolation plates developed various types of bacteria actinomycetes and fungal colonies. Colonies selected from each plate were four to five based on colony appearance. Colonies observed at first, second and third days were eliminated because actinomycetes are considered as slow grower (Ariffuzzaman et al. 2010). Pure isolates grew well on the Kusters agar media forming well isolated colonies. Twenty selected isolates were examined microscopically and identified by their morphological and culture characteristics. Colonies having characteristic features such as powdery appearance and color ranging from white, gray to pinkish and yellowish were selected. The isolates obtained formed colored tough, leathery and filamentous colonies that were hard to pick from the culture media (Plates 1-5), as a characteristic of the genus Streptomyces and also produced colored pigments, which were secreted into the culture media within a few days of incubation. 


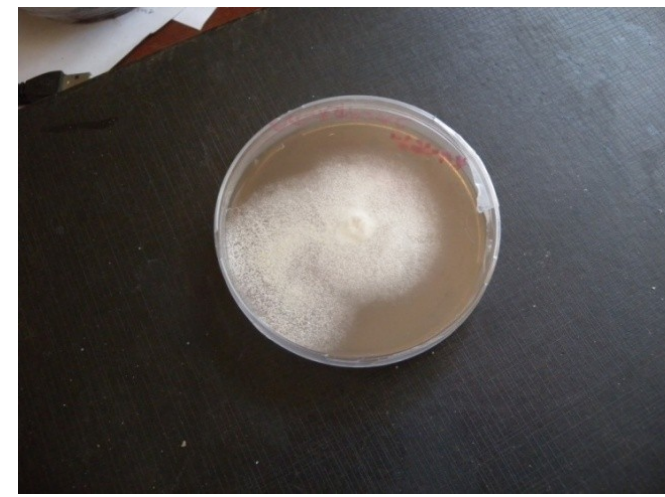

Plate 1: AT 5 isolate

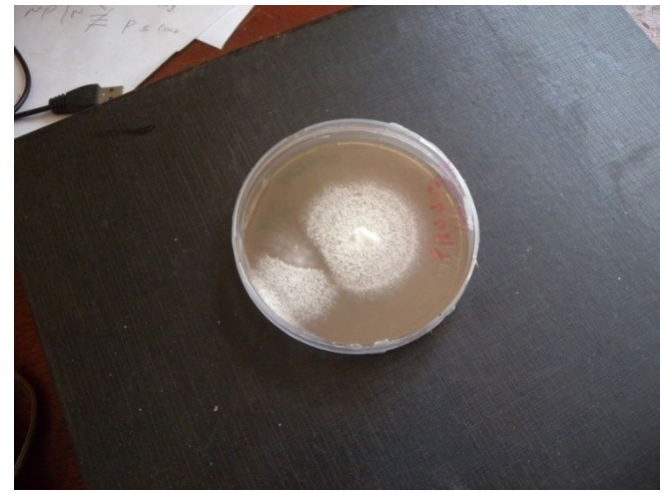

Plate 2: AT 2 isolate

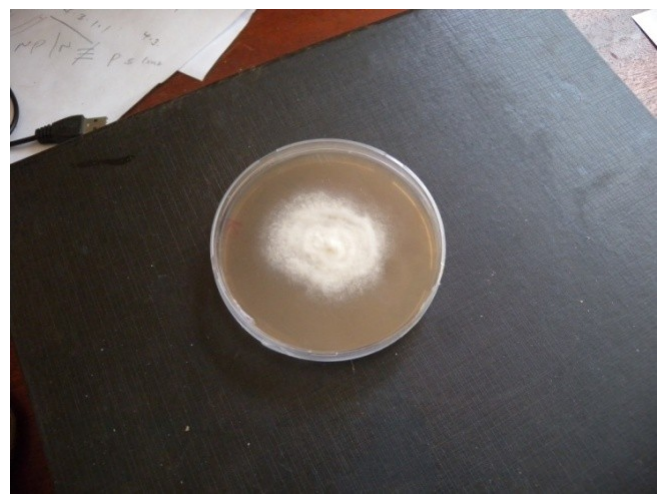

Plate 2: AT 4 isolate

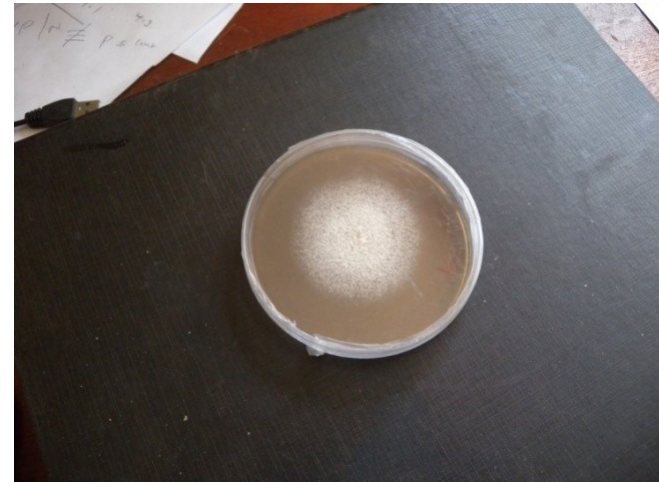

Plate 4: AT 3 isolate

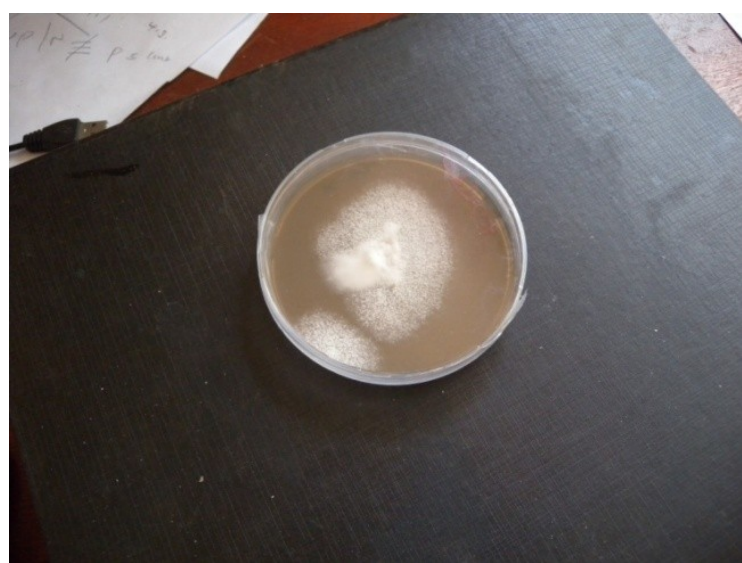

Plate 5: AT1 isolate

\subsection{Pyricularia grisea isolated}

Pyricularia grisea was easily isolated on autoclaved finger millet debris; however its conidiation is slow. It has a good growth on PDA and easily produced conidia under fluorescent light. It produces white mycelium that forms concentric rings on the growth medium (Plate 6). The ascospores are spear-shaped with a tail like structure at the posterior end. 


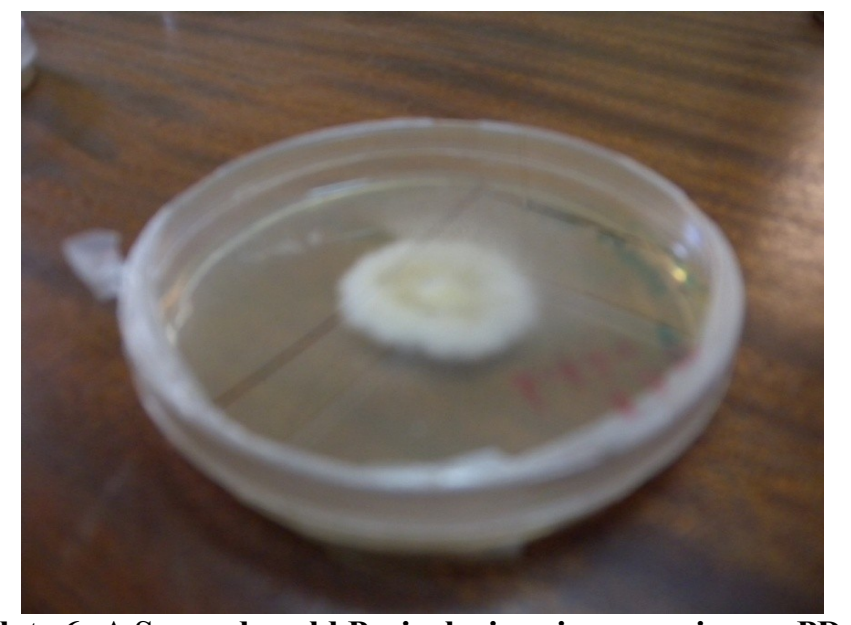

Plate 6: A Seven day old Pyricularia grisea growing on PDA

\subsection{Invitro observations}

Two out of the five Streptomyces isolates screened, showed moderate inhibition (AT2 and AT4 in table one).These results are captured in table one, figure one and experimental plates. It also confirms the studies of Zarandi et al (2009) and Ningthoujam et al (2009). The observation made confirms the candidacy of some strains of Streptomyces in the bio-control of the Pyricularia fungus.

3.3.1 Inhibition zone in ( $\mathrm{mm}$ ) of five Streptomyces isolates against Pyricularia grisea

"Table 1" shows a summary of the results obtained when all the five isolates were plated at a zone of $20 \mathrm{~mm}$. These recordings were made after every 24 hours and tabulated.

\begin{tabular}{|l|l|l|l|l|l|}
\hline \multirow{2}{*}{ Isolate } & \multicolumn{7}{|c|}{ Table 1 } \\
\cline { 2 - 7 } & 24 & 48 & 72 & 96 & 120 \\
\hline AT1 & 16 & 8 & 6 & 4 & 2 \\
\hline AT2 & 17 & 14 & 11 & 10 & 10 \\
\hline AT3 & 18 & 10 & 6 & 3 & 0 \\
\hline AT4 & 15 & 10 & 8 & 7 & 7 \\
\hline AT5 & 14 & 5 & 2 & 0 & 0
\end{tabular}

Figure 1: Inhibition zone for five Streptomyces isolates against P. grisea

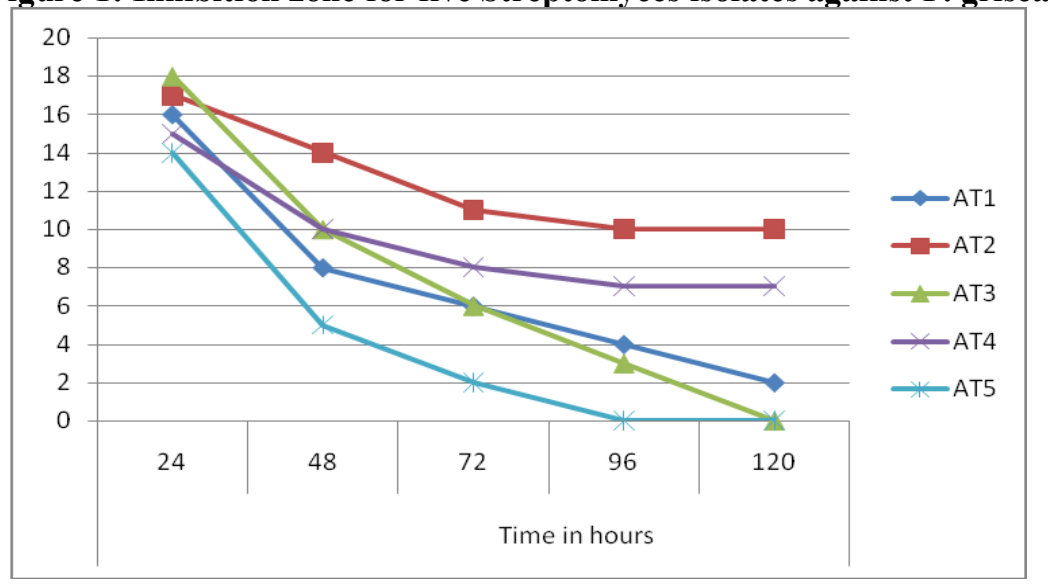

NB; Y axis; Inhibition zone in millimeters

$\mathrm{X}$ axis; Time in hours

\subsubsection{Growth Inhibition rates.}

At the end of $5^{\text {th }}$ day, "Plate 7" (AT1) showed a low inhibition rate with a zone of $2 \mathrm{~mm}$ ("Table 1 "), while the growth inhibition observed in "Plates 8 and 10" (i.e. AT2 and AT4) showed some resistance. "Plates 
9\&11" (AT3 and AT5) had the weakest effect at $0 \mathrm{~mm}$, a fact that is also visible when you look at the recordings in "Table 1").

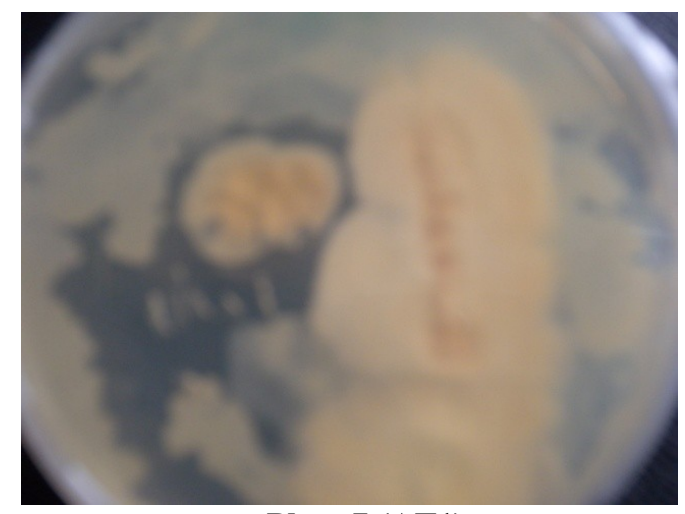

Plate 7 (AT1)

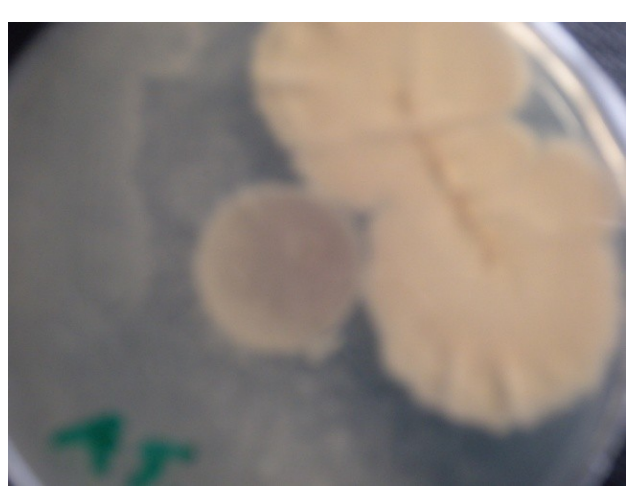

Plate 9 (AT 3)

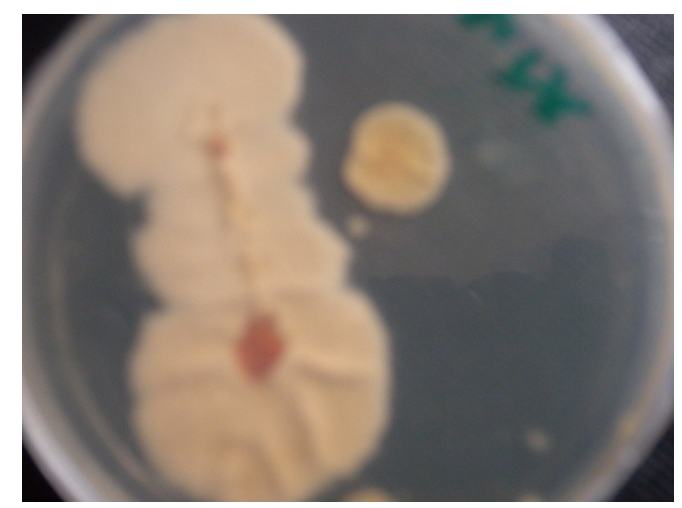

Plate 8 (AT 2)

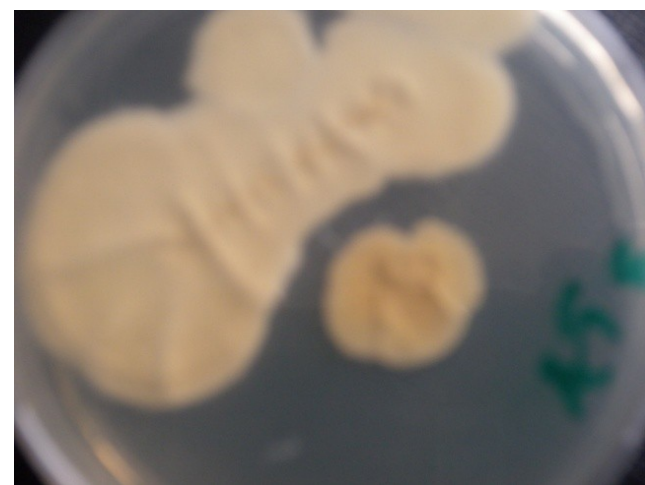

Plate 10 (AT 4)

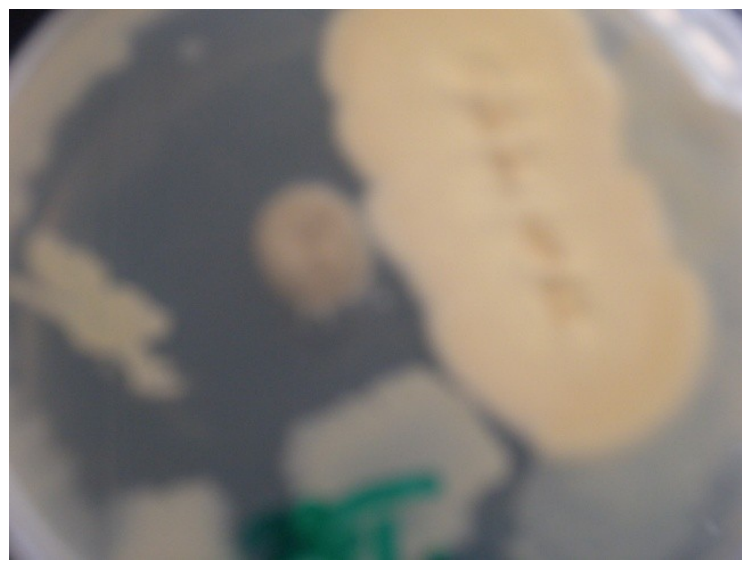

Plate 11 (AT 5)

\section{Conclusions And Recommendations}

We are glad to report the findings of this study. We have established here that Maseno soils are rich in a wide range of Streptomycetes strains (AT1-AT5). Some of these strains (AT 2) have the potential to inhibit Pyricularia grisea growth in a medium (PDA). The ongoing work is to establish if the candidate(AT2) has some useful metabolites that can be used to manage the pathogen it its natural host(finger millet).

Further studies are necessary to isolate more strains within this region and other poor countries with the aim of coming up with strong candidates for bio-control. Harvesting metabolites from these microbes for carrying out analysis on their chemical composition and active ingredients for industrial production has now become clearly a prioriy. More research needs to be done to come up with a suitable formulation of actinomycetes for field application as a cheap long term self sustaining biopesticide in poor third world countries in Africa, Asia and the Latin America. 


\section{Acknowledgement}

We are grateful to the Maseno University, School of physical and biological sciences and the Department of Botany for making this work possible. We also wish to be thankful to the following personalities for their support, Prof. S. O. Wagai for his review and positive comments. Special thanks go to; Daniel Buyela, Solomon and Ms Emma all of the Department of Botany, Maseno University.

\section{References}

[1]. Oduori Chrispus and Kanyenji B. "Finger Millet in Kenya: Importance, Advances in R\&D, Challenges and Opportunities for Improved Production and Profitability." Finger millet blast management in East Africa. International Crops Research Institute for the Semi-Arid Tropics (ICRISAT) (2007) pg 10-22.Print

[2]. Takan J.P. "Pathogen diversity and management of finger millet blast in East Africa: A summary of project activities and outputs." International Sorghum and Millets Newsletter 45(2004): Pg 66-69.Print.

[3]. Mgonja M.A., Manyasa E., Kibuka J., Kaloki P., Nyaboke S. and Wandera G . "Finger Millet in East Africa:Importance, Blast Disease Management and Promotion of Identified Blast Resistant Varieties in Western and Nyanza Provinces of Kenya." Finger millet blast management in East Africa. . (2007): Pg 49-64.Print.

[4]. Sreenivasaprasad S., Takan J.P., MuthumeenakshiS., ObilanaA.B, Manyasa E.O, AudiP.O., Oduori C.A, Coll R.,BrownA.E, NJ Talbot5 and Bandyopadhyay R. "Finger Millet Blast in East Africa: Pathogen Diversity and Disease Management." Finger millet blast management in East Africa. International Crops Research Institute for the Semi-Arid Tropics (ICRISAT), (2007): pg 3445.Print.

[5]. Talbot,N. J. "On the trail of a cereal killer: Exploring the biology of a Magnaporthe grisea." Annual Review of Microbiology. 57(2003): 177-202.Print

[6]. Hong-Sik Oh and Yong-Hwan Lee. "A Target-Site-Specific Screening System for Antifungal Compounds on Appressorium Formation in Magnaporthe grisea." School of Agricultural Biotechnology and Research Center for New Bio-Materials in Agriculture, Seoul National University, (2000): Suwon 441-744. Print.

[7]. Arifuzzaman M., Khatun M.R., and Rahman H. "Isolation and screening of actinomycetes from Sundarbans soil for antibacterial activity." African Journal of Biotechnology Vol. 9(29) (2010): pp. 4615-4619.Print.

[8]. Nonoh J. O., Lwande W., Masiga D., Herrmann R., Presnail J.K., Schepers E., Okech M.A, Bagine R., Mungai P., Nyende A.B. and Boga H.I. "Isolation and characterization of Streptomyces species with antifungal activity from selected national parks in Kenya." African Journal of Microbiology Research Vol. 4(9). (2007): pp. 856-864.Print.

[9]. Tinatin D,Nurzat T. "Biodiversity of Streptomycetes of high-mountainous ecosystems of Kyrgystan and its biotechnological potential." Antonie Leeuwenhoek, 89(2006): 325-328.Print

[10]. Zarandi M.E., Shahidi Bonjar G.H.,Padasht Dehkaei F.,Ayatollahi Moosavi S.A.,Rashid Farokhi P. and Aghighi S. "Biological Control of Rice Blast (Magnaporthe oryzae) by use of Streptomyces sindeneusis isolate 263 in Greenhouse.” American Journal of Applied Sciences 6 (1). (2009): 194-19Print.

[11]. Dastager SG, Wen-jun Li,Dayanand A., Shu-Kun Tang,Xing-Peng Tian Xiao-Yang Zhi, Li-Hua Xu and Cheng-Lin Jiang. "Separation, identification and analysis of pigment (melanin) production in Streptomycetes." African journal of Biotecnology. Vol. 5(2006): pp 1131-1134.Print.

[12]. Reddi GS, Rao AS. "Antagonism of soil actinomycetes to some soil-borne plant pathogenic fungi." Ind. Phytopathol. 24(1971): 649-657.Print

[13]. Rothrock CS, Gottlieb D. "Role of antibiosis of Streptomyces hygroscopicus var. geldanus to Rhizoctonia solani in soil." Can. J. Microbiol., 30(1984): 1440-1447.Print.

[14]. Baskaran,R., Vijayakumar, R. and Mohan, P.M. "Enrichment method for the isolation of bioactive actinomycetes from mangrove sediments of Andaman Islands, India.” Malaysian Journal of Microbiology, Vol 7(1) 2011(2010): pp. 26-32.Print.

[15]. Ningthoujam S D, Sanasam S, Tamreihao K and Nimaichand S. "Antagonistic activities of local actinomycete isolates against rice fungal pathogens.” African journal of microbiology research. vol.3 (11) 2009; pp.737-742. 